\section{Entorno de práctica profesional en enfermería.}

\section{Estimado editor:}

Un entorno de práctica profesional en enfermería (EPPE) es el que promueve, facilita u obstaculiza al personal de enfermería para prestar cuidados de calidad, además de incrementar la seguridad y bienestar de los pacientes y de sus profesionales. Construir entornos positivos fomenta la excelencia de la organización, optimiza los resultados y mejora la percepción y satisfacción del usuario.

En numerosos países se ha podido constatar que determinados ámbitos asistenciales de enfermería considerados excelentes influyen de forma objetiva en los resultados en salud, con disminución de la morbimortalidad, aumento de la calidad y mejora del cuidado (1), además de la contención de costes (2) y otros múltiples beneficios para la sociedad, el ciudadano, el usuario y el profesional. Elementos del entorno como la calidad del liderazgo en enfermería, la participación de la enfermera en la toma de decisiones, la continuidad del cuidado, la competencia continuada, las relaciones adecuadas entre enfermeras y médicos, la formación y el nivel formativo, y la percepción de una plantilla adecuada, entre otros elementos, se han mostrado claves para mejorar los EPPE y los cuidados prestados.

Estas son razones suficientes que justifican el estudio en profundidad de los EPPE para poder construir entornos de trabajo que ayuden a mejorar el cuidado, la mejora continua, la práctica basada en evidencias y la excelencia.

La mayoría de estudios se han centrado en entornos hospitalarios; la evidencia avala el impacto que pueden tener sobre la salud en los hospitales, pero en la atención primaria de la salud todavía queda pendiente cerrar la brecha existente en cuanto a la evidencia disponible al respecto.

Mejorar los EPPE en el ámbito de la atención primaria de la salud tendrá repercusiones sobre la salud de la comunidad; se ha demostrado que los cuidados domiciliarios de calidad reducen los ingresos hospitalarios (3), con beneficios indirectos al evitar posibles tratamientos costosos y otros riesgos asociados a la hospitalización; por ejemplo, se ha relacionado la calidad del EPPE con el incremento o la reducción de las úlceras por presión en domicilios (4), o como determinante en el riesgo de infecciones y de las buenas prácticas de las cuidadoras en el hogar (5).

¿Son los EPPE en la atención primaria de la salud un elemento influyente en la calidad de los cuidados que presta el personal de enfermería a la población? Proponemos que el estudio, la medición y la mejora de los EPPE en el ámbito comunitario fortalecerán también la salud global de la población en su ambiente vital: la comunidad.

Los EPPE deberían resultar de especial interés para los profesionales de la gestión y administración sanitaria, puesto que como determinantes estructurales que son de la atención a la salud, ayudarán a estos gestores a conocer mejor las fortalezas y debilidades de sus equipos y organizaciones, guiando sus decisiones sobre características a reformar para mejorar la satisfacción y los resultados en salud de la población, y posibilitando que se configuren centros de salud saludables.

Esta última afirmación parece relevante en la medida en que los propios sistemas sanitarios están diseñando estrategias de evaluación y mejora de la atención primaria de la salud y porque, además, los riesgos de la falta de conocimiento sobre los entornos pueden empeorar los resultados en salud, precipitando una mayor morbimortalidad así como un incremento de eventos adversos y complicaciones no deseados.

Modificar los elementos más significativos del entorno organizacional requeriría, posiblemente, escasos recursos económicos, por lo que resultaría muy eficiente en comparación con los beneficios resultantes. La faceta económica parece especialmente atractiva dentro del clima de desinversión en los sistemas sanitarios derivada de la crisis financiera mundial y los procesos privatizadores. Además, aumentar la evidencia en el ámbito de la atención primaria de la salud aportaría herramientas contundentes para reclamar dichos recursos esenciales que faciliten tales resultados, que podrían ayudar a tener profesionales más satisfechos e implicados con el sistema de salud.

Vicente Gea-Caballero

Escuela de Enfermería La Fe (centro adscrito Universidad de Valencia), Valencia, España.

Enrique Castro-Sánchez

Raúl Juárez-Vela

Universidad San Jorge de Zaragoza, Zaragoza, España

Carmen Sarabia-Cobo

Universidad de Cantabria, Santander, España

Miguel Ángel Díaz-Herrera Institut Català de la Salut, Barcelona, España.

José Ramón Martínez-Riera

Universidad de Alicante, Alicante, España

Forma de citar Gea-Caballero V, Castro-Sánchez E, JuárezVela R, Sarabia-Cobo C, Díaz-Herrera MA, Martínez-Riera JR. Entorno de práctica profesional en enfermería. Rev Panam Salud Publica. 2018;42:e48. https:/ / doi.org/10.26633/RPSP.2018.48

Este es un artículo de acceso abierto distribuido bajo los términos de la licencia Creative Commons Attribution-NonCommercial-NoDerivs 3.0 IGO, que permite su uso, distribución y reproducción en cualquier medio, siempre que el trabajo original se cite de la manera adecuada. No se permiten modificaciones a los artículos ni su uso comercial. Al reproducir un artículo no debe haber ningún indicio de que la OPS o el artículo avalan a una organización un producto específico. El uso del logo de la OPS no está permitido. Esta leyenda debe conservarse, junto con la URL original del artículo. 


\section{REFERENCIAS}

1. You L, Aiken LH, Sloane DM, Liu K, He G, Hu Y, et al. Hospital nursing, care quality, and Patient satisfaction: cross-sectional surveys of nurses and patients in hospitals in China and Europe. Int J Nurs Stud 2013;50(2):154-161.

2. Chuan-Fen Liu, Nancy D. Sharp, Anne E. Sales, Elliott Lowy, Matthew L. Maciejewski, Jack Needleman, et al. Line Authority for Nurse Staffing and Costs for Acute Inpatient Care. Inquiry 2009;46(3):339-351.

3. Jarrín O, Flynn L, Lake ET, Aiken LH. Home health agency work environments and hospitalizations. Med Care 2014;52(10):877-883.
4. Flynn L, Liang Y, Dickson GL, Aiken LH. Effects of nursing practice environments on quality outcomes in nursing homes. J Am Geriatr Soc 2010;58(12):2401-2406.

5. Gershon RR, Pogorzelska M, Qureshi KA, Stone PW, Canton AN, Samar SM, et al. Home Health Care Patients and Safety Hazards in the Home: Preliminary Findings. In: Henriksen K, Battles JB, Keyes MA, Grady ML, editors. Advances in Patient Safety: New Directions and Alternative Approaches (Vol. 1: Assessment). Rockville: Agency for Healthcare Research and Quality; 2008. 